\title{
Cross-Correlations of the Forex Market Using Power Law Classification Scheme Picture
}

\author{
J. MIŚKIEWICZ ${ }^{a, b, *}$ \\ ${ }^{a}$ Institute of Theoretical Physics, University of Wrocław, pl. M. Borna 9, 50-204 Wrocław, Poland \\ ${ }^{b}$ Department of Physics and Biophysics, Wrocław University of Environmental and Life Sciences, C.K. Norwida 25, \\ 50-375 Wrocław, Poland
}

\begin{abstract}
The analysis of crisis influence on the cross-correlation of the foreign exchange market (forex) daily exchange rates time series is presented. The analysis was conducted on 42 exchange rates with PLN as the common base currency. The time series cover the period from 09.10.2007 till 08.08.2015. Cross-correlation of the time series was analysed by power law classification scheme. It was shown that the strength of correlation allows not only to properly distinguish crisis and prosperity periods but also, followed by network analysis, is capable of recognizing the nodes which are the source of the crisis.
\end{abstract}

DOI: 10.12693/APhysPolA.129.917

PACS/topics: 89.65.Gh, 05.45.Tp

\section{Introduction}

Globalization of the world economy does not only stimulate the economic growth [1], improve food security and resource sustainability $[2]$, and obviously influence politics [3], but also results in increase of cross-correlation among financial time series [4-7]. The growth of mutual dependences among various entities (firms, companies, bonds, shares, currencies, etc.) become a subject of interest of econophysicists, who were developing methods of cross-correlation analysis e.g. [8-12]. The recently developed methods can be grouped into three main branches. The first set consists of the methods based on the variance of modified signals DCCA [13] and their modifications $[14,15]$. The second group of methods are the algorithms originated in DFA such that MF-CCA $[8,16]$. The third set, very promising, are the Manhattan distance based algorithms - the entropy analysis $[6,7]$ and the power low classification scheme (PLCS) [11, 12, 17, 18]. The later method allows measuring the strength and stability of correlations. It was shown that the strength of correlation properly distinguishes and classifies crises among stock markets [18]. In the present analysis the time series of the forex market are analysed - namely the exchange rates of polish zloty to 42 currencies in the interval from 09.10.2007 till 08.08.2015 (2000 data points). The detailed list is presented in Sect. 3. The main aim of this analysis is to verify whether the PLCS algorithm can be applied to the analysis of the forex market data. It is expected to observe crises on the country (currency) level, on the very sensitive and liquid market. Additionally, it is verified if the strength of correlation matrix allows distinguishing "source" of the crisis.

*e-mail: janusz.miskiewicz@ift.uni.wroc.pl
The paper is organised as follows: Sect. 2 shortly summarises basic features of PLCS, Sect. 3 presents the analysed data. The results and conclusions are presented in Sects. 4 and 5 , respectively.

\section{Power law classification scheme}

The cross-correlation of the forex market daily exchange time series are analysed by PLCS. The method was introduced in [11] and later developed in [12] and [17]. Although the detailed description of the algorithm can be found in [11, 12,17], for the convenience of the reader the main steps of PLCS are recalled also here. The algorithm is based on the Manhattan distance. Let us denote analysed time series by $A$ and $B$ and its element as $a_{i}$ and $b_{i}$, respectively. Then the Manhattan distance (MD) is defined as:

$$
M D(A, B)(j)=\sum_{i=1}^{j}\left|a_{i}-b_{i}\right|, j \in\{1,2, \ldots, T\} .
$$

$T$ is the number of data points in the time series. Observe that the value of MD (Eq. (1)) depends on the time series length. So the cumulative MD, such that

$$
\begin{aligned}
& \{M D(A, B)(1), M D(A, B)(2), M D(A, B)(3), \ldots, \\
& \quad M D(A, B)(T)\},
\end{aligned}
$$

is not trivial. Then one can present the cumulative MD series as a function of $j$ in $\log -\log$ scale and fit linear function to the data. The correlation strength $\alpha$ is defined as the slope coefficient of the fitted linear function $(\gamma)$ decreased by one i.e. $\alpha=\gamma-1$. The stability of correlation $\beta$, the second PLCS parameter, is defined as the probability of linear hypothesis rejection given by the $F$-test in the analysis of variance (ANOVA).

Although the range of possible cases is very broad, for interpretation purposes it is worth to consider few typical examples: (i) if the time series $A$ and $B$ are converging 
i.e. $\left(\forall i \in N,\left|a_{i}-b_{i}\right|>\left|a_{i+1}-b_{i+1}\right|\right)$ then $\alpha<0$, (ii) for synchronous time series (and linearly correlated) i.e. $\left(\forall i \in N,\left|a_{i}-b_{i}\right|=\left|a_{i+1}-b_{i+1}\right|\right) \alpha=0$, (iii) in the case of slowly diverging (the diverging time series are such as $\left.\left.\forall i \in N, \mid a_{i}-b_{i}\right)|<| a_{i+1}-b_{i+1} \mid\right) 0<\alpha<1$, (iv) strength of correlation of linearly correlated but diverging time series $\alpha=1$ and (v) for strongly diverging time series $\alpha>1$. The interpretation depends also on the correlation stability parameter. If the model rejection probability is very high then the interpretation of correlation strength is questionable.

\section{Data}

The exchange rates of polish zloty to 42 world currencies in the period from 09.10.2007 till 08.08.2015 are analysed. The following exchange rates are considered here: PLNARS, PLNAUD, PLNBGN, PLNCAD, PLNCHF, PLNCLP, PLNCNY, PLNCZK, PLNDKK, PLNEGP, PLNEUR, PLNGBP, PLNHKD, PLNHRK, PLNHUF, PLNIDR, PLNILS, PLNINR, PLNISK, PLNJPY, PLNKRW, PLNMXN, PLNMYR, PLNNAD, PLNNOK, PLNNZD, PLNPHP, PLNRON, PLNRUB, PLNSEK, PLNSGD, PLNTHB, PLNTRY, PLNTWD, PLNUAH, PLNUSD, PLNXAG, PLNXAU, PLNXDR, PLNXPD, PLNXPT, PLNZAR. Polish zloty plays the role of the common base currency. The abbreviations follow the standard forex market notation. Besides the currencies, also the exchange rates of goods playing the role of currencies in a crisis such as gold, silver or platinum are included in the analysis.

The time series were normalised by transforming them into logarithmic returns $R(t)$ :

$$
R(t)=\ln \left(\frac{p_{t}}{p_{t-1}}\right) .
$$

$p(t)$ denotes the exchange rate at time $t$. Due to a large number of analysed time series there will only be presented plots of the average and standard deviation of the ensemble of $\log$ returns as a function of time. In Fig. 1 the crisis in 2009 year can be recognised as the increase of range of mean value of $R(t)$ as well as the local maximum of standard deviation of $R(t)$. However, taking into account that the evolution of mean of $R$ is rather scattered the recognition of crises requires additional historical knowledge.

\section{Results}

The analysis of crises signatures on the forex market time series was performed by analysing the evolution of correlation strength between the chosen time series, which was reconstructed by moving time window technique. According to observation made in previous analyses $[11,12,17,18]$ two time window sizes were chosen: $T=50 \mathrm{~d}(\mathrm{~d})$ and $T=100 \mathrm{~d}$. The analysis was conducted using overlapping time windows, so the time window was shifted by one day at each step and the PLCS algorithm applied. Since the log-return time series consisted
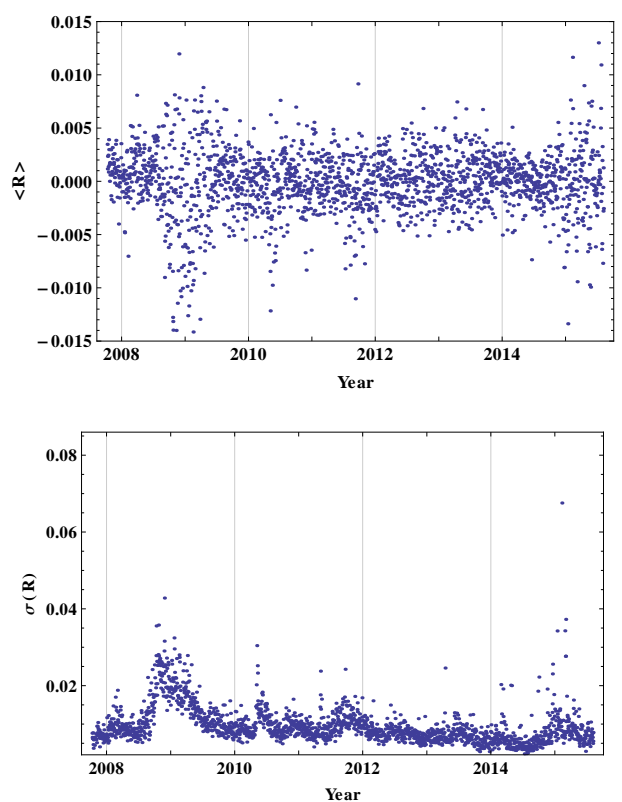

Fig. 1. Evolution of average and standard deviation of daily logarithmic returns of exchange rates of PLN to the chosen set of currencies.
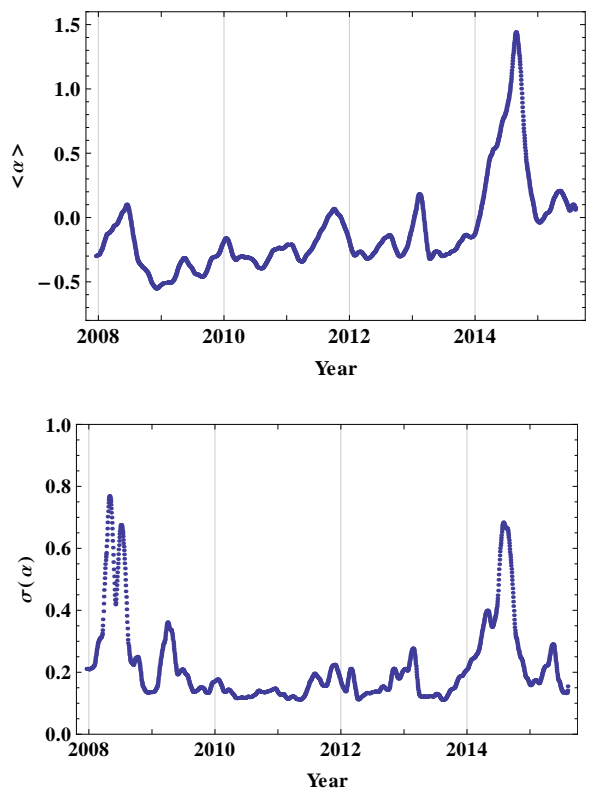

Fig. 2. The time evolution of the mean value and standard deviation of the correlation strength matrices. The time window $T=50 \mathrm{~d}$.

of 1999 data points the moving time window technique transformed it into 1950 correlation strength matrices for $T=50 \mathrm{~d}$ and 1900 for $T=100 \mathrm{~d}$. Moreover, each of the matrices consists of 861 independent elements above (or below) diagonal. Therefore, it is necessary to apply matrix analysis to be able to reach conclusions. The main aims of the analysis are to observe what are the crisis effects on the forex market in PLCS analysis and indicate the most vulnerable to the crisis as well as the most robust currencies. Therefore, to following characteristics are calculated: 

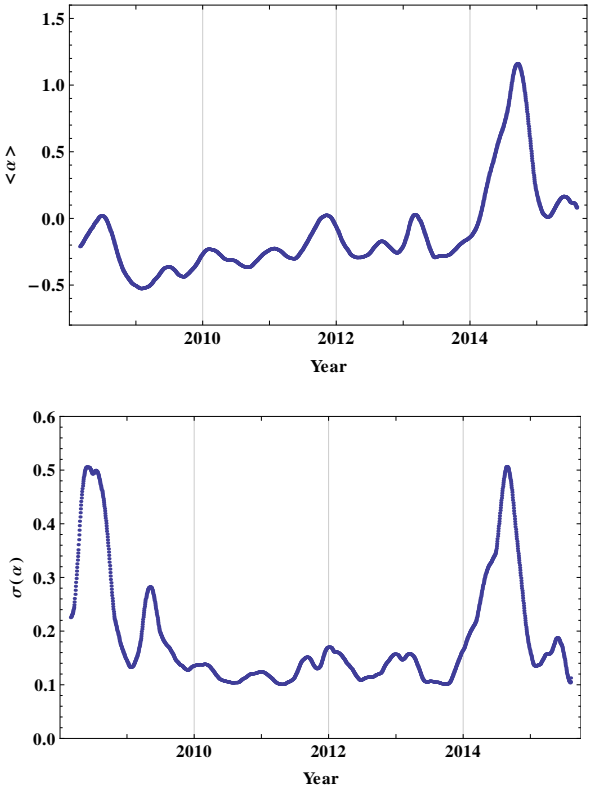

Fig. 3. The time evolution of the mean value and standard deviation of the correlation strength matrices. The time window $T=100 \mathrm{~d}$.

- the crisis effects on the whole system are investigated by the analysis of the mean value and the standard deviation of the correlation strength matrix,

- while the potentially vulnerable (and robust) currencies are searched by constructing the strength preferential network and performing analysis of its structure.

\subsection{Crisis recognition}

Analysis of stock market indices [18] indicates that the crisis can be recognised by the maxima of the time evolution of the mean value and standard deviation of the correlation strength matrices. In Figs. 2 and 3 the maxima of average and standard deviation of the correlation strength can be easily recognised and related to the observed crises. Particularly well seen are the maxima of 2009 and 2015 years, with the observation that the averaged strength of the correlation was lower in 2009 year than in 2015 year. It is due to the fact that at 2009 the crisis was caused by the situation on the stock market and had a smaller impact on forex market while in 2015 year the crisis was related to the money market, moreover, the "centre" of the crisis which was in Europe so stronger affected the exchange rates of PLN. The stability of correlation of calculated models was $\beta<1.2 \times 10^{-3}$ for $T=50 \mathrm{~d}$ and $\beta<3.5 \times 10^{-7}$ for $T=100 \mathrm{~d}$.

\subsection{Crisis centres}

The structure of time series dependences was investigated by constructing strength preferential network. The nodes of the network are connected if the correlation
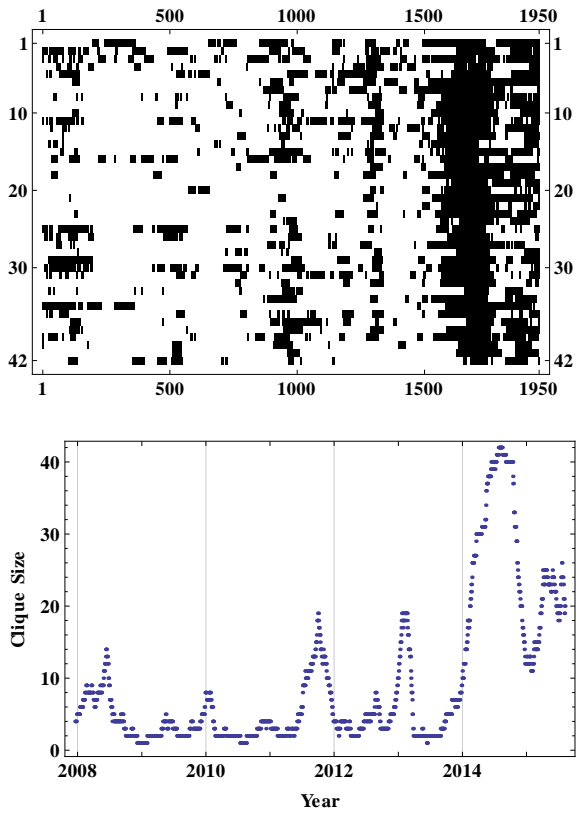

Fig. 4. Clique members (top). The number on the vertical axis corresponds to the position of the exchange rate on the list in Sect. 3. The consecutive number of the analysed time window is presented on horizontal axis. The exchange rates series being the members of a clique are represented by the black square. Evolution of the clique size in time (bottom). The time window $T=50 \mathrm{~d}$.

strength between appropriate time series is $\alpha>0$. In a similar way the complementary network i.e. similarity preferential can be constructed, but then the nodes are connected if $\alpha<0$. For the strength preferential network, the nodes are connected when their correlation strength is greater than zero, which can be interpreted as diverging time series so the differences between the considered time series are increasing. The increase of differences among times indicates usually a crisis, particularly when it is observed at the same time between several nodes $[11,12,17,18]$. Analysing the crisis level one usually looks for the set of the most affected nodes. Therefore, firstly the cliques are considered, so the group of currencies which are, in some sense, the most affected by the crisis. The graphs illustrating the cliques members and size of the clique in time are shown in Fig. 4. The names of the exchange rates were replaced by the position of the exchange rate on the list presented in Sect. 3 . The time series being the member of a clique is denoted by the black square. The crisis of 2009 year was recognised in the mean correlation strength plots of Figs. 2 and 3, as the first maximum in cliques analysis in Fig. 4 is observed as the first maxima of clique size. It is worth noticing that this crisis was relatively long and on the graph illustrating the participation of an exchange rate in a clique the time period of relatively stable clique is around 200 data points, which is quite long comparing with the cliques formed in crisis 2011 year and 2013 year which lasted about 80 and 50 data points, respectively. The last crisis observed in 2014 year resulted in the for- 


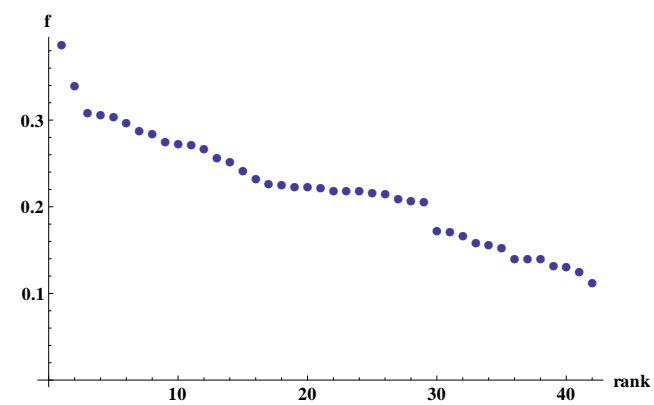

Fig. 5. The rank plot of the being the clique member frequency of the strength preferential network. The time window size $T=50 \mathrm{~d}$.

mation of fully connected graphs and could be considered as the most severe for all exchange rates of PLN, probably due to the situation in Ukraine.

Analysing the graph of the clique members in Fig. 4 one could speculate that some of the currencies are more frequent cliques members than the others, so the corresponding economies might be more vulnerable to crisis occurrence. Therefore, the frequency analysis of clique members was performed. The frequency of being the clique member is defined as the number of networks where the considered time series is the member of a clique to the total number of time windows.

The rank plot being the clique member frequency is presented in Fig. 5.

The first exchange rate in the rank is the PLNARS. It coincides with the recent financial problems of Argentina. The next point of the rank is PLNBGN and reflects the situation in Bulgaria. The two next exchange rates might be slightly surprising because these are PLNCHF and PLNEUR, but considering the fact that European Community (and its currency) was recently under the pressure of financial markets due to the situation in Greece, Portugal and even in Italy the position of EUR is perfectly understood. The same is with CHF, which was under such a strong pressure that Swiss National Bank decided to introduce the minimum threshold of EURCHF exchange rate. The fifth and sixth position in the rank is occupied by PLNCLN and PLNUAH exchange rates. Considering the recent, very difficult situation in Ukraine the position of PLNUAH is fully justified.

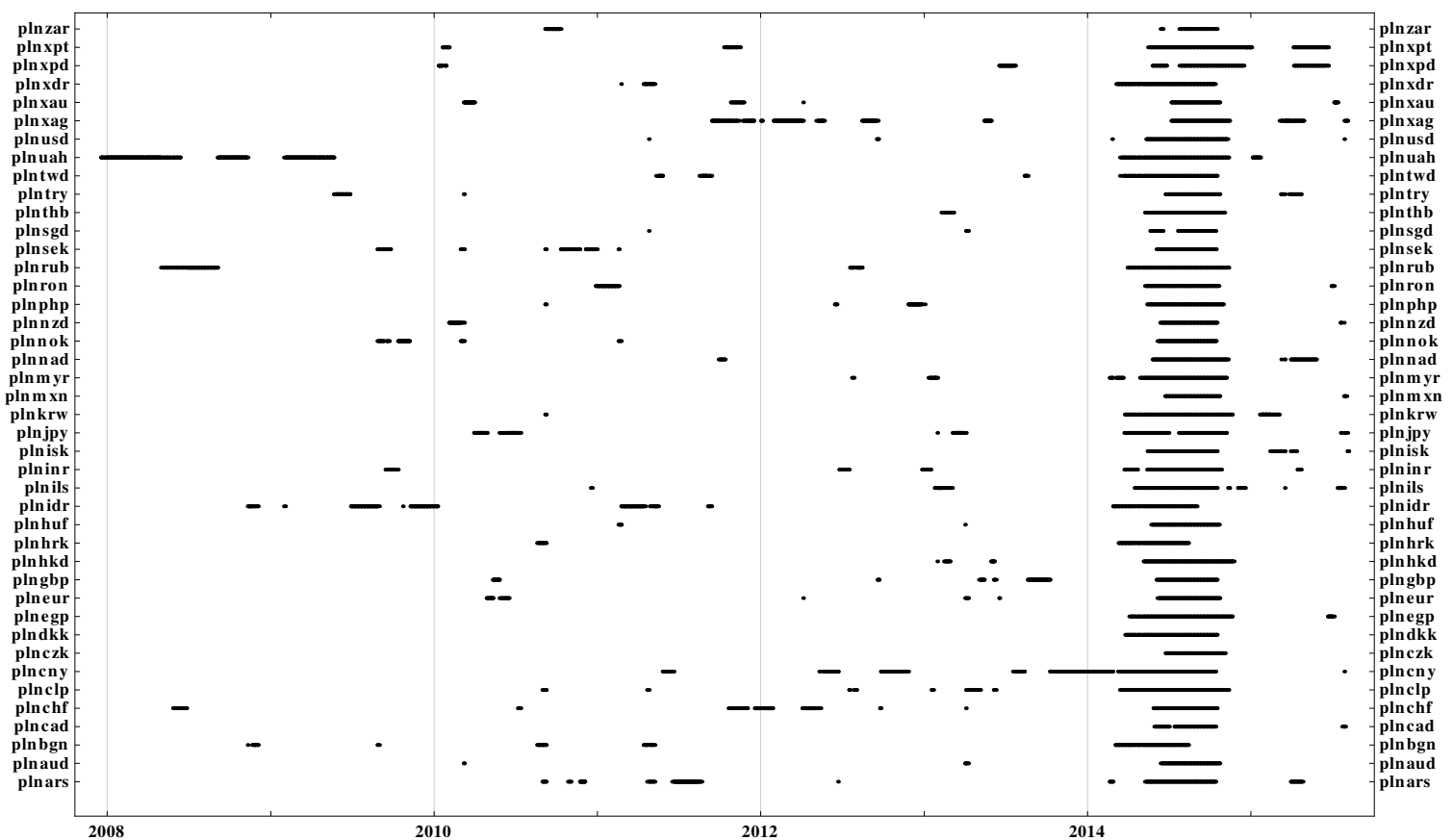

Fig. 6. The main hubs of the strength preferential networks constructed on the basis of the correlation strength matrices of analysed exchange rates time series. Time window $T=50 \mathrm{~d}$.

Continuing the analysis of the strength preferential network the main hubs of the network were investigated. The results are presented in Fig. 6. Since strength preferential network does not impose constrains on the network structure it may happen then there are several nodes with the highest number of links. In fact, such a situation is observed quite often. The most spectacular case was in the middle of 2015 year, where the fully connected graph was observed. Although, a single occurrence of being the main hub of the strength preferential network may indicate problems but the situation when the currency is the main hub of the network for several consecutive time windows shows that the currency is under strong pressure. Although, the list of currencies being under a 
serious stress is quite long the discussion is restricted to the most important cases. For example, the exchange rate PLNUAH was the main hub in 2008 and the first half of 2009 year, which can be related to the political crisis in Ukraine. The second example discussed here is the time series of PLNCHF, which was the main hub at the end of 2011 year and beginning of 2012 year. This position of Swiss frank is related to the financial crisis and measures which were taken by FED and EBC to protect the USA and European financial systems. From the global point of view, it is very interesting that the Chinese currency takes high position in 2012-2014 years. Since China is one of the globally influencing countries its problems might have a great impact on the global economy. The very special case is that in the middle of 2014 year, the beginning of the hybrid war on Ukraine. This situation strongly affected the neighbouring countries, among them Poland. Therefore, the number of links on the strength preferential network rapidly increased and even fully connected graph was observed.

The results of network structure analysis in the case of the time window $T=100 \mathrm{~d}$ were very similar to the $T=50 \mathrm{~d}$, therefore, the discussion is not repeated.

\section{Conclusions}

In the present world economy, the forex market plays a special role. On the one hand, it allows to money flow necessary for foreign trade, but it is also a place of investing. Therefore, in the globalized world, it reflects the state of the country economy. It should be mentioned that due to its liquidity and extremely high trading frequency the forex market is a very sensitive and precise measure of the economy state. In the present work 42 daily exchange rates with PLN as the common base currency were investigated by PLCS algorithm. As in previously analysed systems $[11,12,17,18]$, so in the case of the forex market, the maxima at the plots of the evolution of the mean value and standard deviation of the correlation strength matrix allow distinguishing crises. In difference to the stock market, where a single share or index is investigated in the forex market, the pair of currencies is the subject of the analysis, so it introduces "a point of view" related to the choice of the base currency. Therefore, it is possible to observe the situation when the base currency is under pressure. Then the fully connected graph is observed in the case of the strength preferential network. It is shown that the clique analysis can be applied in distinguishing the potential crisis centres. Particularly, the frequency of being the member of a clique point out the currencies which are the non-incidental members of the clique. The clique analysis conclusions might be supported by the search of the hubs of the strength preferential networks. It was shown that the currencies being hubs in several consecutive time windows were currencies of the country under serious economical crisis. The additional advantage of the analysis is that it might be performed practically in real time giving the investors information about the potential crisis centres.

\section{References}

[1] G.M. Grossman, E. Helpman, Am. Econ. Rev. 105, 100 (2015).

[2] G.K. MacDonald, K.A. Brauman, Shipeng Sun, K.M. Carlson, E.S. Cassidy, J.S. Gerber, P.C. West, BioScience 65, 275 (2015).

[3] E. Owen, D.P. Quinn, Brit. J. Polit. Sci. 46, 95 (2016).

[4] J. Miśkiewicz, M. Ausloos, Int. J. Mod. Phys. C 17, 317 (2006).

[5] J. Miśkiewicz, M. Ausloos, Physica A 387, 6584 (2008).

[6] J. Miśkiewicz, M. Ausloos, Physica A 389, 797 (2010).

[7] J. Miśkiewicz, Physica A 387, 6595 (2008).

[8] R. Rak, S. Drożdż, J. Kwapień, P. Oświęcimka, Euro Phys. Lett. 112, 48001 (2015).

[9] B. Podobnik, D.F. Fu, H.E. Stanley, P.Ch. Ivanov, Eur. Phys. J. B 56, 47 (2007).

[10] V. Plerou, P. Gopikrishnan, B. Rosenow, L.A. Nunes Amaral, H. E. Stanley, Phys. Rev. Lett. 83, 1471 (1999).

[11] J. Miśkiewicz, Physica A 392, 2150 (2013).

[12] J. Miśkiewicz, Acta Phys. Pol. A 123, 589 (2013).

[13] B. Podobnik, H.E. Stanley, Phys. Rev. Lett. 100, 084102 (2008).

[14] Wei-Xing Zhou, Phys. Rev. E 77, 066211 (2008).

[15] Xi-Yuan Qian, Ya-Min Liu, Zhi-Qiang Jiang, B. Podobnik, Wei-Xing Zhou, H.E. Stanley, Phys. Rev. E 91, 062816 (2015).

[16] P. Oświęcimka, S. Drożdż, M. Forczek, S. Jadach, J. Kwapień, Phys. Rev. E 89, 023305 (2014).

[17] J. Miśkiewicz, in: Strength of Cross-Correlations in Crisis. Evolving Network Analysis, Eds.: I. Rojas, G. Ruiz, Copicentro Granada S.L. University of Granada, Spain 2014, p. 269.

[18] J. Miśkiewicz, Acta Phys. Pol. A 127, A-103 (2015). 